\title{
Treatment of odontogenic cutaneous sinus tract misdiagnosed for 6 years
}

\author{
Ben Rjeb Haifa ${ }^{1,2}$, Kallel Ines ${ }^{1,2 *}$, Lejri Walid ${ }^{1,2}$ and Douki Nabiha ${ }^{1,2}$ \\ ${ }^{1}$ Department of Dental Medicine, Sahloul Hospital, Sousse, Faculty of Dentistry, Monastir, Tunisia \\ ${ }^{2}$ Laboratory of Research in Oral Healh and Maxillo Facial Rehabilitation (LR12ES11), Faculty of Dental Medicine, University of Monastir, Monastir, Tunisia
}

\begin{abstract}
Cutaneous odontogenic fistulas or sinus tracts are frequently misdiagnosed and incorrectly treated, leading to unnecessary procedures and patient suffering. An understanding of the draining of cutaneous sinus tracts will lead to more appropriate treatment [1]. Most cases respond to conservative, nonsurgical root canal therapy. Our objective is to report a case of cutaneous sinus tract evolving for 6 years secondary to chronic periapical dental infection caused by old trauma. The conservative treatment of the causal teeth was sufficient to achieve healing despite the oldness of the fistula.
\end{abstract}

\section{Introduction}

An orocutaneous sinus tract is a communication between the oral cavity and the skin surface that is infectious in origin and allows draining of pus from the oral cavity onto the skin surface. The most common location is a tract that originates in the jawbone (usually mandible). A review of case reports shows that after misdiagnosis of this lesion topical and surgical therapy are frequently attempted on the cutaneous aspect of the lesion and no dental treatment is provided. The clinical case reported in this paper emphasizes the need of a prompt recognition of the etiological factor for a correct treatment planning on facial cutaneous sinus tract.

\section{Case report}

A healthy26 -year-old woman presented to our dental department complaining of a non-healing fistula, and a swelling on her chin with an associated purulent discharge (Figure 1). She reported a 6-years history of a drained and recurrent abscess in his face and has been previously treated with different therapeutic procedure for this condition without response :

- The fistula was treated many times with antibiotics prescribed by the dermatologist, who diagnosed the fistula as a furuncle.

- She also underwent two surgical excisions in the maxillofacial department followed by antibiotic treatment without any improvement and the fistula reappears each time.

At the interview the patient reveals the notion of an old traumatism at the age of 15 years.

At the intra oral examination we note a dyschromia of 31 and 41 (Figure 2) both teeth were free of decay but responds negatively to the sensitivity test, the necrosis of teeth 31 and 41 can be explained by the notion of untreated old trauma at the young age.

Endodontic treatment was done to teeth 31 and 41 followed by filling with calcium hydroxyde. A dental radiograph revealed diffuse radiolucency at the apex (consistent with a chronic periapical abscess) (Figure 3).
A check-up appointment is given to the patient after 15 days: No clinical improvement of the lesion, fistula is steel productive (Figure 4).

The lesion was disinfected with Chlorhexidine $0.2 \%$ (Figure 5) and a locating cone was made to check the causative tooth by means of a capillary tips in which we inserted a cone of gutta percka (Figure 6).

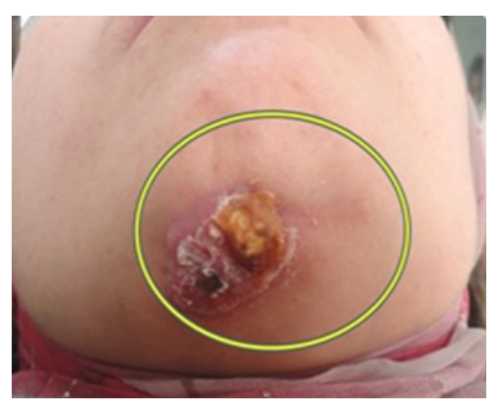

Figure 1. Aspect of an infectedulceration.

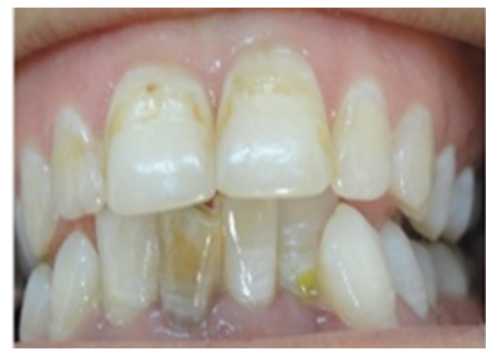

Figure 2. Dyschromia of 41,31 .

Correspondence to: Kallel Ines, Department of Dental Medicine, Sahloul Hospital, Sousse, Faculty of Dentistry, Monastir, Laboratory of Research in Oral Healh and Maxillo Facial Rehabilitation (LR12ES11), Faculty of Dental Medicine, University of Monastir, Monastir, Tunisia, E-mail: ineskallel@yahoo.fr

Received: January 06 2017; Accepted: January 20, 2017; Published: January 24, 2017 


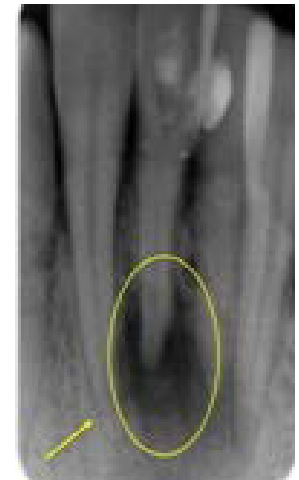

Figure 3.Endodontictreatment of teeth 31 and 41 :filledwith $\mathrm{CaOh} 2$ mixed with Clona (Note the periapicallesion)

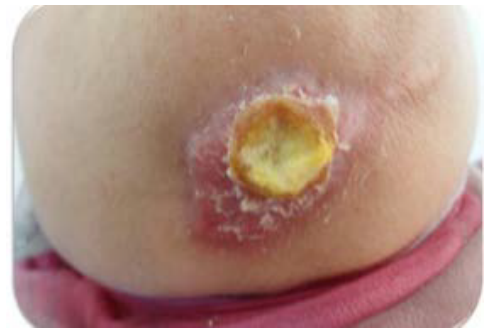

Figure 4. 15-day control: no improvement.

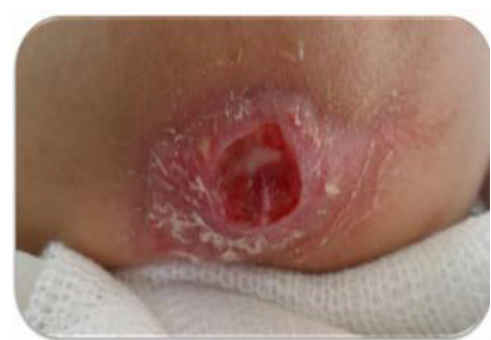

Figure 5. Disinfection of cutaneouslesion.

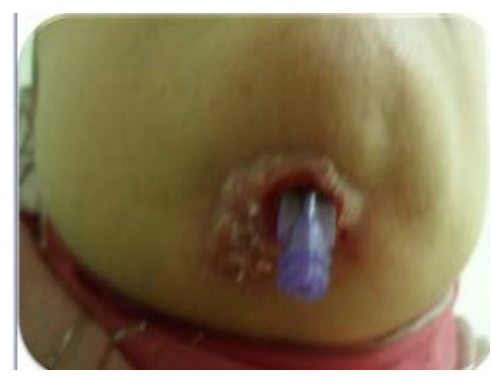

Figure 6. Locating cone with capillary tips in witch gutta percha.

The radiography shows the extension of the periapical image to the 42 (Figure 7).

We decide to be more radical and to treat both the 42 and the 32 (Figure 8), especially we have a radiological examination in 2D (retroalveolar) and the patient does not have the means to make a cone beam to better verify the extension of the periapical lesion.

A control appointment is given after 15 days; we note a slight improvement: the fistula is less productive (Figure 9).

The disinfection and canal sealing was renewed with calcium hydroxide mixed this time with an oily carrier (moru liver oil) to

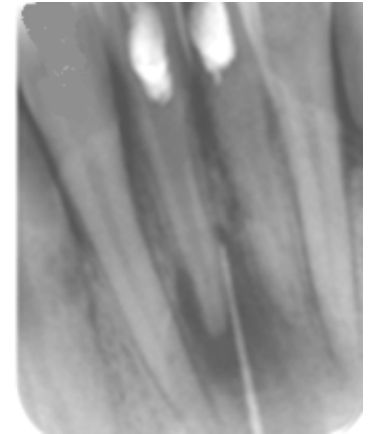

Figure 7. Extension of the periapicallesion to 42 .

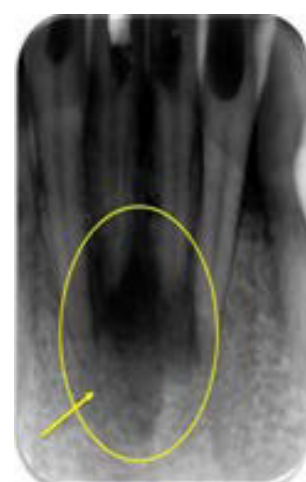

Figure 8. Endodontic treatment of teeth 32,42 and filling with $\mathrm{CaOH} 2$ mixed with Clona.

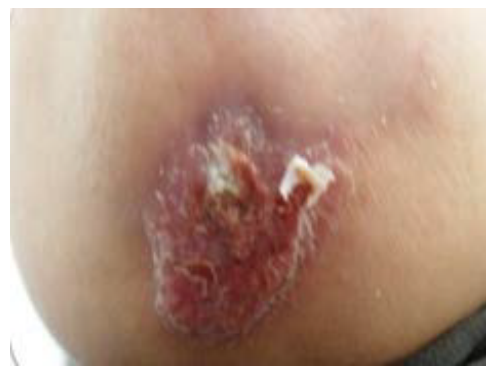

Figure 9. After one month : Slightimprovement of the skin lesion; Renewal of the $\mathrm{CAOH} 2$ mixed with an oily carrier (oil of liver of moru).

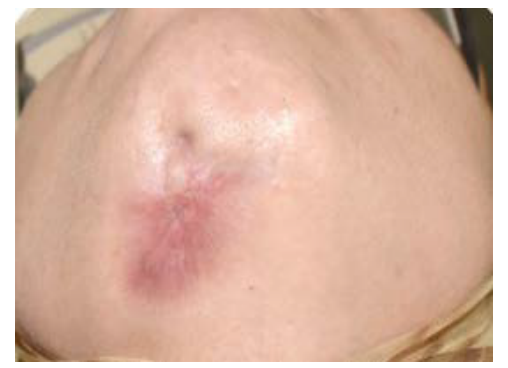

Figure 10. At 2 months: fistulais no longer productive.

prolong the effect of $\mathrm{CaOH}_{2}$ and a control appointment was given one month later.

At 2 months control appointment fistula is no longer productive (Figure 10), no serosity was found in the canal, the majority of the calcium hydroxide was not resorbed, we decide the final root canal filling (Figure 11). After 6 months, the infection resolved, the cutaneous lesion healed and a small umbilication in the skin remained (Figure 12).

The radiological examination show a bone healing (Figure 13). 


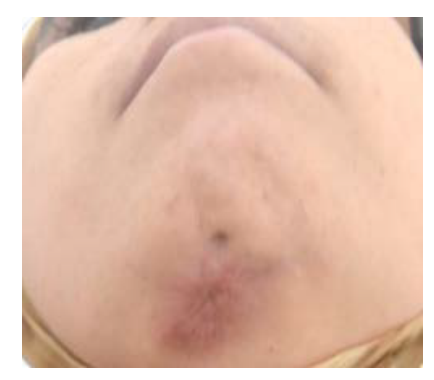

Figure 12. Clinical control 6 monthslater:good evolution.

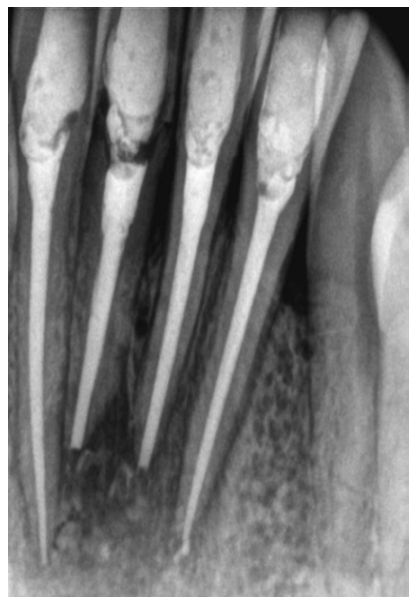

Figure 13. Radiological control 6 monthslater: Bonehealing.

\section{Discussion}

\section{Localisation}

Making the diagnosis of a draining sinus tract in the setting of a chronic dental infection can be challenging for several reasons. The cutaneous lesions do not always arise in close proximity to the underlying infection, and only about half of all patients ever recall having had a toothache [2]. The sinus tracts appear most commonly on the chin or jaw line, but they can also appear elsewhere on the face or neck. Lesions have even been reported to occur as far away from the oral cavity as the chest, thigh, or sacrum. This finding can be explained by the fact that chronic process slowly evolves through the cancellous alveola bone, following the path of least resisitance until it perforate the cortical plate of the mandible [3]. Odontogenic cutaneous sinus tract have a greater likelihood to occur than intraoral in the apices of the teeth are superior to the maxillary muscle attachments or inferior tothe mandibular muscle attachments [4].

\section{Differential diagnosis}

Many patients seek evaluation from several physicians before an accurate diagnosis is made,since the cutaneous lesions can mimic other disorders, such as basal cell carcinoma, pyogenic granuloma, foreign body reaction, fungul infection, osteomyelitis or furuncle [5].

\section{Inappropriate treatment}

Several inappropriate surgeries and courses of antibiotics are commonly used before definitive therapy is instituted; this is what happened with our patient Who consulted his dermatologist who prescribed him different antibiotic cures and underwent surgical excision in the maxillofacial department before he was been referred to our department.
It has been estimated that half of all patients undergo multiple surgeries and trials of antibiotics before definitive diagnosis. Such diagnostic and therapeutic misadventures highlight the importance of communication between medical subspecialists and general dentistry practitioners in the evaluation of patients with head and neck lesions [6].

\section{Positive diagnosis and treatment}

Odontogenic cutaneous sinus tract can be caused by periapical infections around the root apices resulting from pulp necrosis, caries, pericoronitis or traumatic injury. Radiographic finding are important for diagnosis. On the assumption that the relevent tooth cannont be ascertained by periapical radiography, the tracking of the fistula can guide the ultimate diagnosis . Despite sometimes this radiography remains insufficient to evaluate the extent and the relations of the periapical lesion with the adjacent teeth as in our case where the radiographs in three dimensions find their indications such as the cone beam. This fact can explain the absence of healing after the first treatment in which 42 and 32 were not suspected.

Eradication of the original source of infection by means of nonsurgical root canal treatment (if the tooth can be preserved) is most important for treatment of odontogenic cutaneous sinus tract sometimes complemented by surgery.

Automatic closure of the tract should be anticipated within 7 to 14 days after root canal treatment [5]. In this case, the reduction of the abscess and the closure of sinus tract was obtained after 2 months.

This finding can be explained by the delay in identifying causal teeth and by the oldness of the lesion. For this reason, we choosed at the third appointement to renew the calcium hydroxide mixed with an oily carrier (Oil of liver of moru) to prolong the action of calcium hydroxide (decreasing acidity, inflammation and intracanal serosities). Sometimes surgical excision of the cutaneous sinus is nececssary to elimnate the inflammatory tissu (such us apical debridement, apicoectomy, fistula debridement)

In our case no tissu debridement was needed since at 2 months appointement fistula is no longer productive so the root canal treatment validated the primary dental origin of the skin lesion and definitive root canal filling was done.

At 6 months control appointment the lesion on the chin recovered entirely leaving only a slight hyperpigmented region, and the periapical lesion disappeared on the radiographic examination.

\section{Conclusion}

Because patients with cutaneous facial sinus tracts of dental origin often do not have obvious dental symptoms, a possible dental etiology may be overlooked. If dental origin is suspected, the diagnosis is easily confirmed by dental examination and dental radiography of the involved area. Early correct diagnosis, based on radiologic evidence of a periapical root infection and treatment of these lesions can help prevent unnecessary and ineffective antibiotic therapy or surgical treatment, reducing the possibility of further complications.

\section{References}

1. Gime'nez-García, Fernando Martinez-Vera, Leticia Fuentes-Vera (2015) Cutaneous sinus tracts of odontogenic origin: two case reports rosa. J Am Board Fam Med 28: 838-840. [Crossref]

2. Talita Ribeiro Tenório de França, Flávia Maria de Moraes Ramos-Perez, José Divaldo Prado, Danyel Elias da Cruz Perez (2014) Nasal sinus tract associated with dental 
infection. Ann Dermatol 26: 115-116. [Crossref]

3. Peermohamed S, Barber D, Kurwa H (2001) Diagnosic challenges of cutaneous draining sinus tracts of odontogenic origin: a case report. Dermatol Surg 37: 15251527. [Crossref]

4. Gupta M, Das D, Kapur R, Sibal N (2011) A clinical predicament-diagnosis and differential diagnosis of cutaneous facial sinus tracts of denatl origin: a series of case report. Oral Surg Oral Med Oral Pathol Oral Radiol Endod 112: 132-136. [Crossref]

5. Liang (2016) Diagnosis treatment of odontogenic cutaneous sinus tracts in a 11 year old -boy: a case report. Medicine 95: 20.

6. Sheehan DJ, Potter BJ, Davis LS (2005) Cutaneous draining sinus tract of odontogenic origin: unusual presentation of a challenging diagnosis. South Med J 98: 250-252. [Crossref]

Copyright: $\mathbb{0} 2017$ Allam E. This is an open-access article distributed under the terms of the Creative Commons Attribution License, which permits unrestricted use, distribution, and reproduction in any medium, provided the original author and source are credited. 